\title{
Direct photon emission from a chemically non-equilibrated quark-gluon plasma at finite chemical potential
}

Yogesh Kumar*

Department of Physics and Astrophysics, University of Delhi, New Delhi, India E-mail: vogesh.du81damail.com

\section{S. Somorendro Singh}

Department of Physics and Astrophysics, University of Delhi, New Delhi, India E-mail: Sssinghephysics.du.ac.in

We extend to evaluate electromagnetic radiation using modified quark mass from a chemically non-equilibrated quark-gluon plasma with finite baryonic density. In this study, the total photon rate is computed upto two loop processes incorporating parametrization factor in both the quark mass and coupling value. The photon spectrum is obtained by integrating the photon rate over the space-time history of the plasma. The result is found enhancement in the photon emission by the presence of chemical potential and parametrization factor in the quark mass and coupling value. The result shows valuable insights in the measurement of photon production.

XXII. International Workshop on Deep-Inelastic Scattering and Related Subjects, 28 April - 2 May 2014

Warsaw, Poland

\footnotetext{
* Speaker.
} 


\section{Introduction}

The significant experiments of ultra-relativistic heavy-ion collisions performed at RHIC (BNL) and LHC (CERN) have indicated the creation of hot and dense medium of quarks, antiquarks and gluons called the quark-gluon plasma (QGP) [W, [, []]. It is believed that the creation of QGP exist only for a short duration of time and its direct detection is very difficult in these experiments. There are various indirect signatures for its detection, each of which has its advantages and disadvantages. Among all signatures, photons and dileptons are considered as one of the best signal to probe the properties of QGP. Fortunately, the mean free path of photons in the fireball is much larger than its dimensions [ [⿴囗十, 国]. So photon provide a unique and direct probe of the fireball. At high temperature and finite chemical potential, it is expected that the system is in QGP phase due to collision of massive nuclei. The experiments at AGS and SPS energies have already assumed the formation of QGP by considering the chemical potential. So, the collision between two massive nuclei is not fully transparent and it has some finite value of chemical potential. This type of assumption of existing chemical potential is also not completely ignored by the RHIC energies [6]. The information lead to the modification of photon emission rates at finite chemical potential. As examples, Hammon, Geiger and their co-workers [ [ $[$, 8$]$ have indicated that the initial QGP system produced at the RHIC energies has finite value of chemical potential. Traxler et al. have calculated the photon rate of a QGP at finite quark chemical potential for a given temperature [Q]. Biro, Strickland and their co-workers [एU, [U] studied the photon emission in a chemically equilibrating baryon free QGP system.

Thus our work focuses on the photon radiation in a chemically non-equilibrated QGP system with finite chemical potential at 1-loop and 2-loop to reveal the effect of the quark chemical potential on photon production. The performed calculations are parametrically suited using thermal quark mass for RHIC energy with respect to longitudinal expansion of the plasma. The longitudinal expansion is reasonably good for the choice of high temperature. However, we ignore the electromagnetic radiation produced by the transverse direction. Thus, the total photon yield is calculated by integrating the rate over the plasma volume created by the expansion.

In this article, we use a simple model of quark-gluon plasma in which thermal quark mass is generated due to the interaction among quarks and gluons. The thermal dependent quark mass is defined as Kumar et al. [ㄴ, 미]:

$$
m_{q}^{2}=\gamma_{q} g^{2}(p) T^{2}
$$

with $\mathrm{T}$ is the temperature and $\gamma_{q}=1 / 9$ is the phenomenological parameter used to take care the hydrodynamical aspects of the hot QGP. The term $g^{2}(p)=4 \pi \alpha_{s}$ with QCD strong coupling constant $\alpha_{s}$ defined as;

$$
\alpha_{s}=\frac{4}{\left(33-2 n_{f}\right) \ln \left(1+\frac{p^{2}}{\Lambda^{2}}\right)}
$$

Using equation (1.1), we calculate the production rate by replacing the factor $T^{2}$ to a good accuracy of temperature as $T^{2}+\mu_{q}^{2} / \pi^{2}$ [四, 国]. This implies that the quark mass is not only depends on temperature but also is a function of chemical potential. Thus quark mass is suitably modified for 
a chemically non-equilibrated plasma with finite chemical potential by using Kumar et al. [[2], [13]:

$$
m_{q}^{2}=\gamma_{q}\left(\lambda_{q}+\frac{\lambda_{g}}{2}\right) \frac{16 \pi}{\left(33-2 n_{f}\right)} \frac{T^{2}}{\ln \left(1+\frac{p^{2}}{\Lambda^{2}}\right)}\left(1+\frac{\mu_{q}^{2}}{\pi^{2} T^{2}}\right)
$$

where $\mathrm{p}$ is known as low momentum cut off with quark flavor $n_{f}, \lambda_{q}$ and $\lambda_{g}$ are the fugacity factors for quark and gluon and $\Lambda$ is QCD parameter. The other parameters are referred as Ref. [ए2], [15]]. Using modified quark mass and coupling value, we study the photon radiation at temperature $T=$ $0.57 \mathrm{GeV}$ for the quark flavor $n_{f}=2$ with the variation of chemical potential. At last, we represent the outputs with other theoretical works.

The paper is organized as follows. In section 2, we outline the photon production from a chemically non-equilibrated QGP with finite chemical potential. In section 3, we present the results and in section 4 , we finally give conclusion.

\section{Photon radiation from a chemically non-equilibrated QGP with finite chemical potential}

Photon productions are interesting probe of the quark-gluon plasma at finite quark chemical potential. There are many works on photon production at finite chemical potential. It is observed that the thermal photon produced from the early phase of QGP which is having high transverse momentum range in which hadronic contributions are negligibly small. Since photons have been proposed as a promising signature of the QGP formation in relativistic heavy-ion collisions, our emphasis lies on the photon production from a non-equilibrated QGP using finite quark chemical potential. In this work, the photon production due to quark-antiquark annihilation process $(q \bar{q} \rightarrow$ $\gamma g$ ) and QCD Compton $(q g \rightarrow q \gamma$ or $\bar{q} g \rightarrow \bar{q} \gamma)$ are considered which appear at one loop level for system of quarks and gluons. The calculation is performed at temperature $T=0.57 \mathrm{GeV}$ for flavor 2 with the Jüttner distribution functions. The Jüttner distribution functions for quarks, anti-quarks and gluons are used as Ref. [Q, प1, प]. We finally obtain the expression of photon radiation for a chemically non-equilibrated QGP at the finite baryon density through annihilation process [1], [4, [5]]:

$$
E \frac{d N^{A n n}}{d^{3} p d^{4} x}=\frac{2 \alpha \alpha_{s}}{\pi^{4}} \lambda_{q} \lambda_{\bar{q}} T^{2} e^{-E / T} \sum_{f} e_{f}^{2}\left[\ln \left(\frac{4 E T}{k_{c}^{2}}\right)-C_{E u l e r}-1\right],
$$

where $k_{c}^{2}=2 m_{q}^{2}$ and $C_{E u l e r}=0.577216$. Similarly, we perform one loop calculation of Compton process $q(\bar{q}) g \rightarrow q(\bar{q}) \gamma$ for a chemically non-equilibrated system at the finite baryon density as [U], [14, [15]:

$$
E \frac{d N^{C o m p}}{d^{3} p d^{4} x}=\frac{2 \alpha \alpha_{s}}{\pi^{4}} \lambda_{q} \lambda_{g} T^{2} e^{-E / T} \sum_{f} e_{f}^{2}\left[\ln \left(\frac{4 E T}{k_{c}^{2}}\right)-C_{E u l e r}+\frac{1}{2}\right] .
$$

Then we study the additional contributions of thermal photon rate at the same order of coupling value $\alpha_{s}$ that is coming from two-loop diagrams of AWS and bremsstrahlung processes. The calculation of these two processes are expressed as [[15]:

$$
E \frac{d N^{A W S}}{d^{3} p d^{4} x}=\frac{2 N_{c} C_{f}}{3 \pi^{5}} \alpha \alpha_{s}\left(\frac{2}{5} \lambda_{q}^{3}+\frac{3}{5} \lambda_{g} \lambda_{q}^{2}\right) \sum_{f} e_{f}^{2} E T e^{-E / T}\left[J_{T}-J_{L}\right],
$$


and

$$
E \frac{d N^{B r e m}}{d^{3} p d^{4} x}=\frac{2 N_{c} C_{f}}{\pi^{5}} \alpha \alpha_{s}\left(\frac{4}{7} \lambda_{q}^{2}+\frac{3}{7} \lambda_{g} \lambda_{q}\right) \sum_{f} e_{f}^{2} T^{2} e^{-E / T}\left[J_{T}-J_{L}\right] \ln (2)
$$

where the value of $J_{T}=1.108$ and $J_{L}=-1.064$ are taken for $n_{f}=2$.

After obtaining the results through all these processes, we again integrate the photon rate over

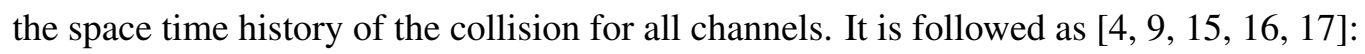

$$
\frac{d N}{d^{2} p_{T} d y}=\int d^{4} x\left(E \frac{d N}{d^{3} p d^{4} x}\right)=Q \int_{\tau_{0}}^{\tau_{c}} \tau d \tau \int d y\left(E \frac{d N}{d^{3} p d^{4} x}\right) .
$$

With the values of rapidity and $p_{T}$, we obtain photon spectra for various channels.
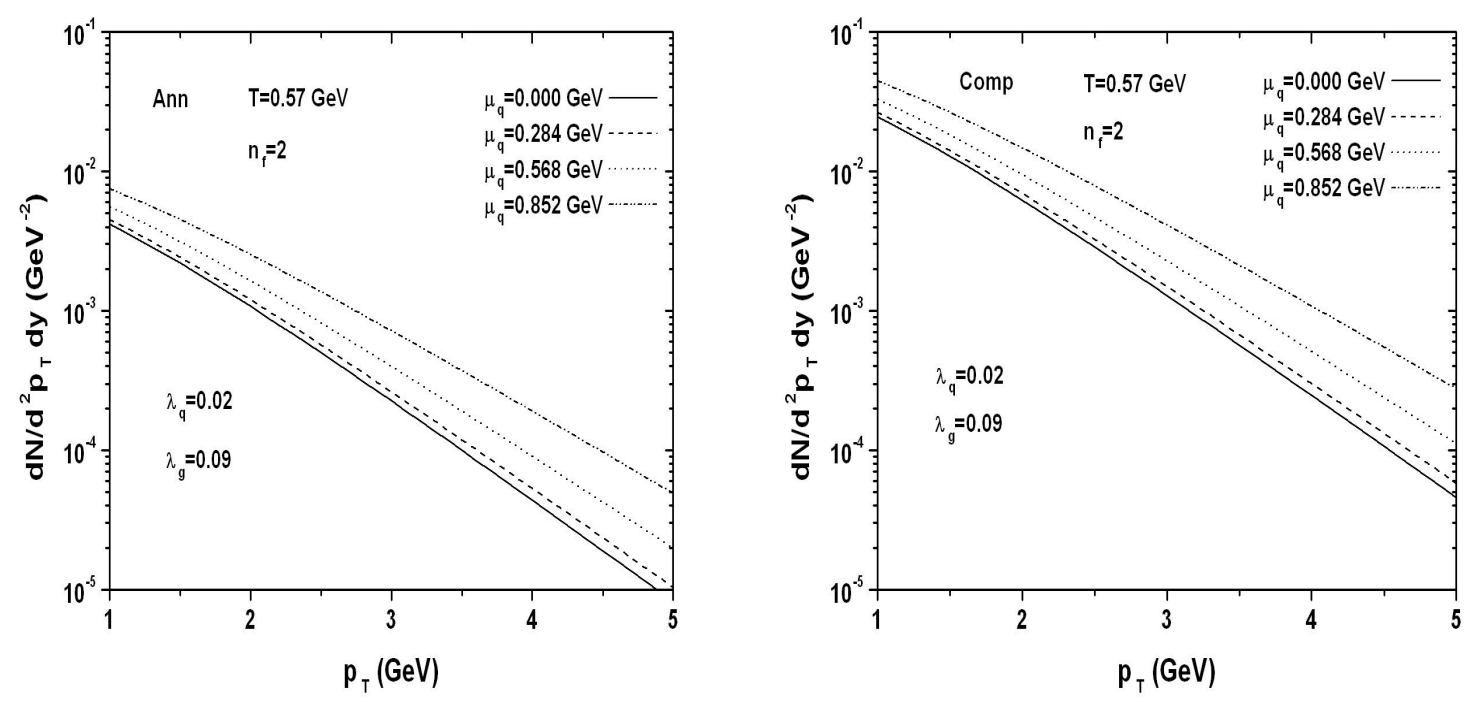

Figure 1: The photon production through annihilation (left) and Compton process (right) are shown at thermal temperature $T=0.57 \mathrm{GeV}$ with the variation of chemical potential for $n_{f}=2$.

\section{Results}

We discuss various production processes of the electromagnetic radiation from a chemically non-equilibrated QGP at finite chemical potential with incorporation of modified quark mass and coupling value. The results show important significance concerning photon production as a signature of quark-gluon plasma. The outputs of photon radiation with the effect of finite quark chemical potential for quark flavor $n_{f}=2$ are discussed as follows.

In Fig. 1 (left) we show the photon production rate at temperature $T=0.57 \mathrm{GeV}$ through the annihilation process for quark flavor $n_{f}=2$. It is found that the output of photon production is strong increasing function of chemical potential $\mu$. We use the temperature $T=0.57 \mathrm{GeV}$ to have a large effect of the thermal photon contribution with increasing the chemical potential. The modified quark mass and coupling value also contribute the enhancement on the production rate 

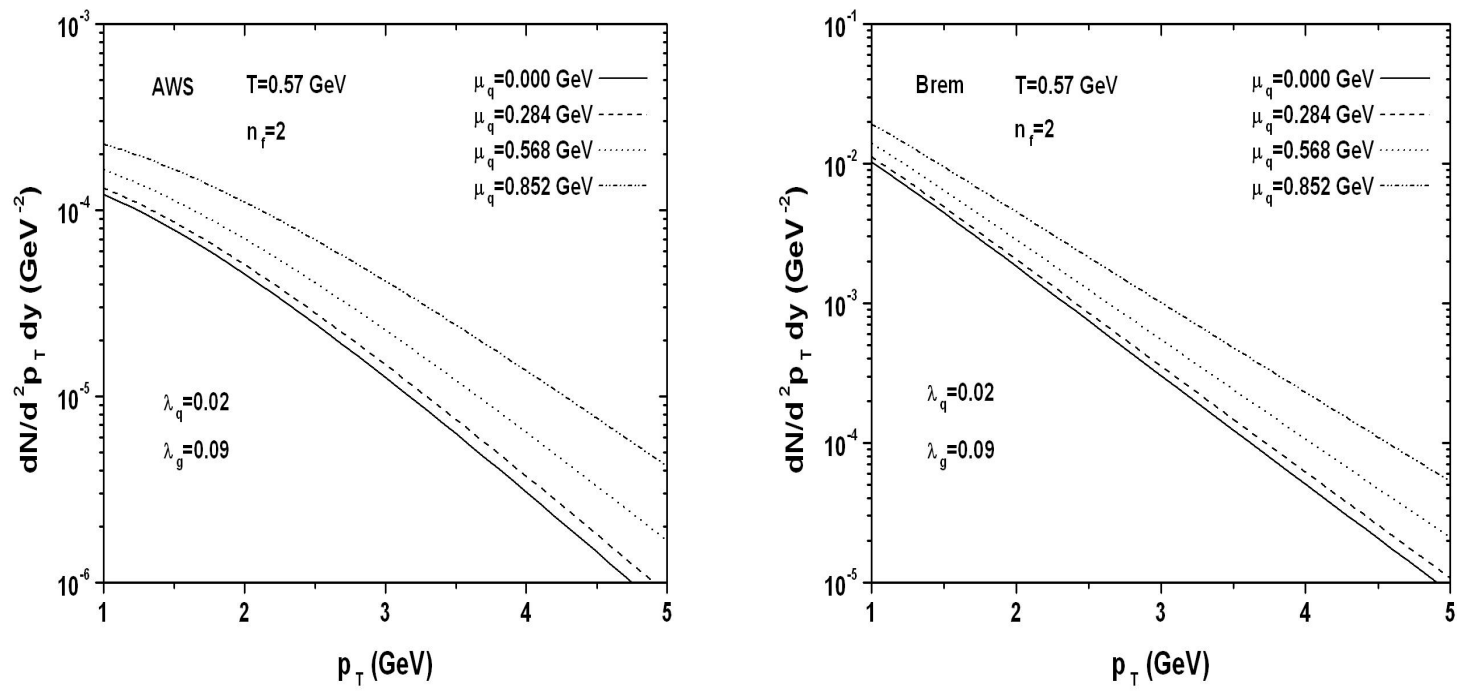

Figure 2: The photon production through annihilation with scattering (left) and bremsstrahlung process (right) are shown at thermal temperature $T=0.57 \mathrm{GeV}$ with the variation of chemical potential for $n_{f}=2$.

and it seems to have a larger production rate at very high temperature and chemical potential. Similarly, in Fig. 1 (right) we look the photon emission at such temperature through the Compton channel. The photon yield is much enhanced in this one loop process. In comparison to annihilation process, the Compton process shows better outcome. This implies that Compton process has more advantage. Thus our results show enhancement in comparison to the other work in the presence of

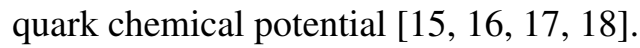

In Fig. 2 (left), we further show the additional contributions to the thermal photon rate at the same order of strong coupling considering two-loop diagram of AWS process. The production rate increases with the effect of various chemical potential but decreases as transverse momentum increases. It is found that AWS channel produce less photons in comparison to the one loop processes of annihilation and Compton during the entire life time of the plasma. This indicates that the production rate produced by AWS process is low in comparison to the other earlier processes. Similarly this type of result is also produced by the experimental data from RHIC. Likewise at LHC, there is indication of strong suppression of the AWS process [प5]]. The huge suppression in the result of AWS process is due to the factor contributed by fugacities which depends cubicly in the production rate. In equilibrium system, the contribution from the AWS process dominates the photon production over other processes but in a chemically non-equilibrated plasma it is suppressed as comparison to other processes. Overall results are less effective in the case of AWS process.

Finally, in Fig. 2 (right), we consider 2 loop level of bremsstrahlung process. Photon production due to this process is large among all previous channels. It is found that the production rate due to bremsstrahlung is less in comparison to the Compton process. In this process also, it is found suppressed result due to fugacity factor. Therefore, in all channels, our result shows dominant behaviour with the influence of parametrization factor with the effect of chemical potential. 
Due to these factors, the model has large advantage in the photon production rate of Compton and bremsstrahlung processes. So the production rate of our model with parametrization factor for flavor $n_{f}=2$ has an improvement from earlier works and give an impact on production rate. The output for photon production rate are compared with the output of Ref. [15, प16, ㅁ], ㅁ]].

\section{Conclusion}

We conclude that the total photon yield is calculated by integrating the rate over the plasma volume created by the expansion. It is well ahead from other theoretical work. The overall calculation of photon production as a function of transverse momentum incorporating the parametrization factor in both the quark mass and coupling value give fine enhanced results from the earlier results without the chemical potential [[3]. So, direct photon emission from a chemically non-equilibrated plasma play important role in the measurement of photon production from high energy heavy-ion collisions at finite chemical potential. It is found to be large increase of production rate in presence of finite chemical potential. Therefore, photon productions are of important signature for giving the signal for the formation of quark-gluon plasma and provide good information for the understanding of QGP.

\section{References}

[1] P. Romatschke, Int. J. Mod. Phys. E 19 (2010) 1; A. Muronga, Phys. Rev. C 69 (2004) 034903.

[2] I. Arsene et al., Nucl. Phys. A 757 (2005) 1; B. B. Back et al., ibid A 757 (2005) 28.

[3] J. Kapusta, P. Lichard and D. Seibert, Phys. Rev. D 44 (1991) 2774; J. Kapusta and L. D. Mclerran, Phys. Lett. B 283 (1992) 145.

[4] T. Peitzmann and M. H. Thoma, Phys. Rep. 364 (2002) 175.

[5] M. H. Thoma, Phys. Rev. D 72 (2005) 094030.

[6] A. Dumitru et al., Mod. Phys. Lett. A 8 (1993) 1291.

[7] N. Hammon et al., Phys. Rev. C 61 (1999) 014901.

[8] K. Geiger and J. I. Kapusta, Phys. Rev. D 47 (1993) 4905.

[9] C. T. Traxler and M. H. Thoma, Phys. Rev. C 53 (1996) 1348; Phys. Lett. B 346 (1995) 329.

[10] T. S. Biro, E. V. Doorn, B. Müller, M. H. Thomas and X. N. Wang, Phys. Rev. C 48 (1993) 1275.

[11] M. Strickland, Phys. Lett. B 331 (1994) 245.

[12] Y. Kumar and S. S. Singh, Can. J. Phys. 90 (2012) 955.

[13] Y. Kumar and S. S. Singh, ISRN HEP 2013 (2013) 156747.

[14] A. K. Chaudhuri, J. Phys. G 26 (2000) 1433.

[15] M. G. Mustafa and M. H. Thoma, Phys. Rev. C 62 (2000) 014902.

[16] D. Dutta, A. K. Mohanty, K. Kumar and R. K. Choudhury, Phys. Rev. C 61 (2000) 064911.

[17] J. Long, Z. He, Y. Ma and B. Liu, Phys. Rev. C 72 (2005) 064907.

[18] J. Long, Z. He, Y. Ma and B. Liu, Nucl. Phys. A 766 (2006) 201. 